\title{
The Probability of a Subspace Swap in the SVD
}

\author{
John K. Thomas, Louis L. Scharf, Fellow, IEEE, and Donald W. Tufts, Fellow, IEEE
}

\begin{abstract}
In this paper we extend the work of Tufts, Kot, and Vaccaro (TKV) to improve the analytical characterization of threshold breakdown in SVD methods. Our results sharpen the TKV results by lower bounding the probability of a subspace swap in the SVD. Our key theoretical result is the characteristic function for a random variable whose probability of exceeding zero bounds the probability of a threshold breakdown.
\end{abstract}

\section{INTRODUCTION}

$\mathbf{T}$ HE singular value decomposition (SVD) is used extensively in high resolution parameter estimation methods. The high resolution of SVD-based methods can be attributed to the splitting of the measurement space into an estimated signal subspace and an orthogonal subspace. However, the performance of SVD methods degrades rapidly when the signal-to-noise ratio (SNR) falls below a threshold SNR. The catastrophic drop in performance is referred to as the threshold effect. Tufts, Kot, and Vaccaro (TKV) [1], [2] associated the threshold effect with the probability that the measured data is better approximated by some components of the orthogonal subspace than by some components of the signal subspace. When this happens, we say a subspace swap has occurred. Our key theoretical result is the characteristic function for a random variable whose probability of exceeding zero bounds the probability of threshold breakdown.

In this paper, we revisit the problem first posed by TKV, namely, the analytical study of threshold effects in linear prediction methods that use the SVD for rank reduction. We improve the results of TKV in two ways:

1) We lower bound the probability of a subspace swap by defining an event that is a subset of the event that a subspace swap occurs. TKV approximated the event that a subspace swap occurs.

2) We compute the exact characteristic function of the random variable that determines our bounding event. TKV approximated the random variable that determines their approximating event by a normal random variable.

With these improvements, we are able to follow the philosophy of TKV and more accurately predict threshold than was previously possible.

Manuscript received June 17, 1993; revised April 4, 1994. This work was supported by the Office of Naval Research, Mathematics Division, Arlington, VA, under contract \# N0001489-J-1070. The associate editor coordinating the review of this paper and approving it for publication was Prof. Kevin M. Buckley.

J. K. Thomas and L. L. Scharf are with the Department of Electrical and Computer Engineering, University of Colorado, Boulder, CO, 80309-0425 USA.

D. W. Tufts is with the Department of Electrical Engineering, University of Rhode Island, Kingston, RI 02881 USA.

IEEE Log Number 9408234.

\section{ANALYSIS OF THRESHOLD}

SVD methods are used to identify the "mode parameters," $\underline{\theta}$, in the signal plus noise model

$$
\mathbf{y}=\mathbf{x}(\underline{\theta})+\mathbf{n} .
$$

Each of the vectors $\mathbf{y}, \mathbf{x}$, and $\mathbf{n}$ is an element of $C^{N \times 1}$. (Notation: $C^{n \times m}$ refers to a matrix of size $n \times m$ of complex numbers.) For example, $\mathbf{x}(\underline{\theta})=\left(x_{1}, x_{2}, x_{3}, \ldots, x_{N}\right)^{T}$, with $x_{n} \in C$. The modal signal $\mathbf{x}(\underline{\theta})$ can be modeled as a sum of damped sinusoids or equivalently as the deterministic impulse response of an ARMA system. The ARMA system can in turn be modeled by its AR and MA coefficients, its poles and zeros, its poles and residues, and so on. Typically, the model for $\mathbf{x}(\underline{\theta})$ is the separable model $\mathbf{x}(\underline{\theta})=\mathbf{H}\left(\underline{\theta}_{1}\right) \underline{\theta}_{2}$, where $\underline{\theta}^{T}=\left(\underline{\theta}_{1}^{T} \underline{\theta}_{2}^{T}\right)$.

The measurement $\mathbf{y}=\mathbf{y}_{r}+j \mathbf{y}_{i}$ is complex, with $\mathbf{y}_{r}=$ $\operatorname{Re}[\mathbf{x}]+\operatorname{Re}[\mathbf{n}]$ and $\mathbf{y}_{i}=\operatorname{Im}[\mathbf{x}]+\operatorname{Im}[\mathbf{n}]$. If the additive noise is assumed to be zero mean, the first two moments of $y$ are

$$
\begin{aligned}
E \mathbf{y} & =\mathbf{x}=\operatorname{Re}[\mathbf{x}]+j \operatorname{Im}[\mathbf{x}] \\
\mathbf{R}_{y y} & =E[\mathbf{y}-\mathbf{x}][\mathbf{y}-\mathbf{x}]^{*}=\mathbf{R}_{n n}=E\left[\mathbf{n} \mathbf{n}^{*}\right] \\
& =E(\operatorname{Re}[\mathbf{n}]+j \operatorname{Im}[\mathbf{n}])\left(\operatorname{Re}[\mathbf{n}]^{T}-j \operatorname{Im}[\mathbf{n}]^{T}\right) \\
& =\mathbf{R}_{11}+\mathbf{R}_{22}+j\left(\mathbf{R}_{21}-\mathbf{R}_{12}\right)
\end{aligned}
$$

where

$$
\begin{aligned}
& \mathbf{R}_{11}=E\left[\operatorname{Re}[\mathbf{n}] \operatorname{Re}[\mathbf{n}]^{T}\right] \\
& \mathbf{R}_{12}=E\left[\operatorname{Re}[\mathbf{n}] \operatorname{Im}[\mathbf{n}]^{T}\right] \\
& \mathbf{R}_{21}=\mathbf{R}_{12}^{T} \\
& \mathbf{R}_{22}=E\left[\operatorname{Im}[\mathbf{n}] \operatorname{Im}[\mathbf{n}]^{T}\right] .
\end{aligned}
$$

When constructing $\mathbf{n}$ from $\operatorname{Re}[\mathbf{n}]$ and $\operatorname{Im}[\mathbf{n}]$, the only theoretical constraint is that $\mathbf{R}_{11}=\mathbf{R}_{11}^{T}>0, \mathbf{R}_{22}=\mathbf{R}_{22}^{T}>0$, and $\mathbf{R}_{12}=\mathbf{R}_{21}^{T}$. In our examples we shall assume

$$
\begin{aligned}
& \mathbf{R}_{11}=\mathbf{R}_{22}=\frac{\sigma^{2}}{2} \mathbf{I} \\
& \mathbf{R}_{12}=\mathbf{R}_{21}=0 .
\end{aligned}
$$

This is the standard complex white noise. That is, $\mathbf{R}_{y y}=\sigma^{2} \mathbf{I}$.

The first step in modal analysis is to argue for the approximate rank deficiency of the Hankel data matrix

$$
\mathbf{Y}=\left[\begin{array}{cccc}
y(1) & y(2) & \cdots & y(m) \\
y(2) & y(3) & \cdots & y(m+1) \\
\vdots & \vdots & \ddots & \vdots \\
y(M) & y(M+1) & \cdots & y(N)
\end{array}\right] \in C^{M \times m}
$$

where the overfitting parameter $m$ exceeds $p$, the dimension of the subspace $\langle\mathbf{X}\rangle$ that contains $\mathbf{x}(\underline{\theta})$. The parameter $M=$ $N-m+1$ is the number of residuals generated from the 
equation $\mathbf{Y a}=\mathbf{u}$. The Hankel structure of $\mathbf{Y}$ produces the representation

$$
\mathbf{Y}=\sum_{n=1}^{N} y_{n} \mathbf{D}_{n}
$$

where $\mathbf{D}_{n}$ is a matrix of zeros and ones, with the ones arrayed on the $n$th super antidiagonal:

$$
\mathbf{D}_{n}=\left[\begin{array}{lll} 
& & \\
& & \\
& & \\
& &
\end{array}\right] \in C^{M \times m} .
$$

The Hankel data matrix $\mathbf{Y}$ contains the signal and noise

$$
\mathbf{Y}=\mathbf{X}+\mathbf{N} ; \quad \mathbf{X}, \mathbf{N} \in C^{M \times m} .
$$

Because the signal $\mathbf{x}$ is modal, its corresponding signal matrix $\mathbf{X}$ has a rank deficient SVD

$$
\mathbf{X}=\mathbf{U} \Sigma_{s} \mathbf{V}^{*}
$$

where the matrices $\mathrm{U}, \Sigma_{s}, \mathbf{V}^{*}$ are defined as follows:

$$
\begin{aligned}
\mathbf{U} & \in C^{M \times M}: \mathbf{U}^{*} \mathbf{U}=\mathbf{I} ; \quad \mathbf{V} \in C^{m \times m}: \mathbf{V}^{*} \mathbf{V}=\mathbf{I} \\
\Sigma_{s} & \in C^{M \times m}: \Sigma_{s}=\left[\begin{array}{cr}
\Sigma_{p} & 0 \\
0 & 0
\end{array}\right] ; \\
\Sigma_{p} & =\operatorname{diag}\left(\sigma_{1}, \sigma_{2}, \ldots, \sigma_{p}\right) ; \quad \sigma_{1} \geq \sigma_{2} \geq \cdots \geq \sigma_{p} .
\end{aligned}
$$

The unitary matrix $\mathbf{U}$ may be partitioned into $\mathbf{U}=\left[\mathbf{u}_{1} \cdots \mathbf{u}_{p} \mid\right.$ $\left.\mathbf{u}_{p+1} \cdots \mathbf{u}_{M}\right]=\left[\mathbf{U}_{p} \mid \mathbf{U}_{0}\right]$, where the "signal subspace" $\left\langle\mathbf{U}_{p}\right\rangle$ contains $\mathbf{X}$ and the "orthogonal subspace" completes $C^{M \times M}$. This is illustrated in Fig. 1 . Now, of course, the data matrix $\mathbf{Y}$ will not lie entirely within the signal subspace $\left\langle\mathbf{U}_{p}\right\rangle$, because it consists of signal plus noise, as illustrated in Fig. 1. However, the projections onto $\left\langle\mathbf{U}_{p}\right\rangle$ and $\left\langle\mathbf{U}_{0}\right\rangle$ resolve $\mathbf{Y}$ into its "signal" and "orthogonal" components, as illustrated in the figure. These are, in fact, least squares estimates of the signal matrix $\mathbf{X}$ and the noise matrix $\mathbf{N}$ :

$$
\begin{aligned}
& \hat{\mathbf{X}}=\mathbf{P}_{\mathbf{U}_{p}} \mathbf{Y}=\mathbf{U}_{p} \mathbf{U}_{p}^{*} \mathbf{Y} \\
& \hat{\mathbf{N}}=\mathbf{P}_{\mathbf{U}_{0}} \mathbf{Y}=\mathbf{U}_{0} \mathbf{U}_{0}^{*} \mathbf{Y} \\
& \mathbf{Y}=\hat{\mathbf{X}}+\hat{\mathbf{N}}=\left(\mathbf{P}_{\mathbf{U}_{p}}+\mathbf{P}_{\mathbf{U}_{0}}\right) \mathbf{Y} .
\end{aligned}
$$

These projections resolve the energy of the data matrix $\mathbf{Y}$ into two components:

$$
\mathcal{E}=\operatorname{tr} \mathbf{Y}^{*} \mathbf{Y}=\operatorname{tr} \mathbf{Y}^{*}\left(\mathbf{P}_{\mathrm{U}_{p}}+\mathbf{P}_{\mathbf{U}_{0}}\right) \mathbf{Y}=\mathcal{E}_{p}+\mathcal{E}_{0}
$$

where

$$
\begin{gathered}
\mathcal{E}_{p}=\operatorname{tr} \mathbf{Y}^{*} \mathbf{P}_{\mathbf{U}_{p}} \mathbf{Y}=\sum_{i=1}^{p} \mathcal{E}_{p i} ; \quad \mathcal{E}_{p i}=\mathbf{u}_{i}^{*} \mathbf{Y} \mathbf{Y}^{*} \mathbf{u}_{i}, \quad 1 \leq i \leq p \\
\mathcal{E}_{0}=\operatorname{tr} \mathbf{Y}^{*} \mathbf{P}_{\mathbf{U}_{0}} \mathbf{Y}=\sum_{i=p+1}^{M} \mathcal{E}_{0 i} ; \quad \mathcal{E}_{0 i}=\mathbf{u}_{i}^{*} \mathbf{Y} \mathbf{Y}^{*} \mathbf{u}_{i}, \\
p+1 \leq i \leq M
\end{gathered}
$$

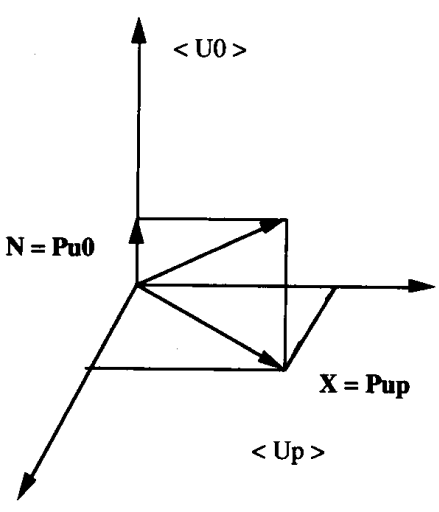

Fig. 1. Signal and orthogonal subspaces.

$\mathcal{E}_{p}$ and $\mathcal{E}_{0}$ are the energies resolved into the respective subspaces, and the $\mathcal{E}_{p i}$ and $\mathcal{E}_{0 i}$ are the energies resolved along the components of $\left\langle\mathbf{U}_{p}\right\rangle$ and $\left\langle\mathbf{U}_{0}\right\rangle$.

In modal analysis, the projections $\mathbf{P}_{\mathbf{U}_{P}}$ and $\mathbf{P}_{\mathbf{U}_{0}}$ are unknown, but the structure of the orthogonal subspace $\left\langle\mathbf{U}_{0}\right\rangle$ is known to be

$$
\left\langle\mathbf{U}_{0}\right\rangle=\langle\mathbf{A}\rangle
$$

where $\mathbf{A} \in C^{M \times(M-p)}$ is the prediction error matrix determined by the prediction error polynomial (PEP) $A(z)=$ $\sum_{i=0}^{p} a_{i} z^{-i}$ :

$$
\mathbf{A}=\left[\begin{array}{ccccccc}
a_{p} & \cdots & a_{p} & 1 & & & \\
& \ddots & & \ddots & & & \\
& & & & & & \\
& & & & \ddots & & \\
& & a_{p} & \cdots & & a_{p} & 1
\end{array}\right]
$$

The zeros of $A(z)$ are the mode parameters $z_{n}$. This observation leads to a host of techniques for identifying the prediction error polynomial (PEP) $A(z)$ and its roots $z_{n}$ [3]-[7]. In all of these procedures, the objective is to identify a subspace $\langle\hat{\mathbf{A}}\rangle$ that is close enough to the subspace $\langle\mathbf{A}\rangle$ such that the corresponding PEP's $A(z)$ and $\hat{A}(z)$ have nearly identical roots. However, this will clearly be impossible if the noise matrix $\mathbf{N}$ is so large that $\mathbf{Y}$ lies far from the true signal subspace $\left\langle\mathbf{U}_{p}\right\rangle$.

One way to measure the distance that $\mathbf{Y}$ lies from the subspace $\left\langle\mathbf{U}_{p}\right\rangle$ is to compare its energy in $\left\langle\mathbf{U}_{p}\right\rangle$ and $\left\langle\mathbf{U}_{0}\right\rangle$ :

$$
\mathcal{E}_{p} \gtrless \mathcal{E}_{0} .
$$

In the noise-free case, $\mathcal{E}_{p}=\operatorname{tr} \mathbf{X}^{\mathrm{X}} \mathbf{X}$ and $\mathcal{E}_{0}=0$, but as the noise increases, $\mathcal{E}_{0}$ increases until a large fraction of the resolved energy lies in $\left\langle\mathbf{U}_{0}\right\rangle$. It was the insight of TKV to recognize that at some point, one or more modes in $\left\langle\mathbf{U}_{0}\right\rangle$ will actually resolve more energy than one or more modes in $\left\langle\mathbf{U}_{p}\right\rangle$. When this happens, important modal information will be swapped between subspaces, making it unavailable for retrieval by a subspace method that uses either the orthogonal subspace or the signal subspace. TKV argued that this 
situation characterizes the performance breakdown associated with threshold effects in modal analysis.

We shall call the situation described above a subspace swap. TKV approximated a swap as the event that $\mathcal{E}_{0}$, the total energy in the orthogonal subspace, exceeds $\sum_{i=p-q+1}^{p} \mathcal{E}_{p i}$, the total energy in $q$ of the a priori least dominant signal modes. But this condition is neither necessary nor sufficient for a swap. On the other hand, the event that the average energy in $\left\langle\mathbf{U}_{0}\right\rangle$, namely, $\mathcal{E}_{0} /(M-p)$, exceeds the average energy in $\left\langle\mathbf{U}_{p}\right\rangle$, namely, $\mathcal{E}_{p} / p$, is sufficient (but not necessary). Therefore, the event

$$
\overline{\mathcal{E}}_{0}=\frac{\mathcal{E}_{0}}{M-p}>\frac{\mathcal{E}_{p}}{p}=\overline{\mathcal{E}}_{p}
$$

characterizes a particular swap event. This event is a subset of the event that one or more modes in $\left\langle\mathbf{U}_{0}\right\rangle$ resolves more energy than one or more modes in $\left\langle\mathbf{U}_{p}\right\rangle$. Why? Because at least one mode in $\mathcal{E}_{0}$ resolves more energy than $\overline{\mathcal{E}}_{0}$ and at least one mode in $\mathcal{E}_{p}$ resolves less energy than $\overline{\mathcal{E}}_{p}$. That is, there exists at least one pair $(i, j)$ such that $\mathcal{E}_{0 i}>\mathcal{E}_{p i}$ whenever condition (15) holds. An alternative characterization of a subspace swap is $\overline{\mathcal{E}}_{0}>\mathcal{E}_{p p}$, where $\mathcal{E}_{p p}$ is the energy resolved into the a priori least dominant mode $\mathbf{u}_{p}$. This alternative characterization is also a sufficient condition for a swap because if it holds then there exists at least one mode $\mathbf{u}_{i}$ in the orthogonal subspace that resolves more energy than the a priori least dominant mode $\mathbf{u}_{p}$.

\section{Probability Bound}

Let $A$ be the event that a subspace swap occurs; $B$ the event that the average energy in the orthogonal subspace exceeds the average energy in the signal subspace; and $C$ the event that the average energy in the orthogonal subspace exceeds the energy resolved along the a priori least dominant signal mode. Then, we know that $A \supset B$ and $A \supset C$, and that $P[A]>P[B]$ and $P[A]>P[C]$. When the signal subspace eigenvalues are clustered, it is unreasonable to associate a subspace swap with the event $C$. Why? Because to isolate $\mathcal{E}_{p p}$ as the only component that can be exceeded is to ignore the probability that one or more of many roughly equal signal subspace energies can be exceeded, and the probability $P[C]$ underestimates $P[A]$. Therefore, when the eigenvalues of the signal subspace are clustered, we choose the event $B$ as the better bounding event. The probability of a subspace swap is bounded below by

$$
P[A] \geq P[B]=P\left[\overline{\mathcal{E}}_{0}>\overline{\mathcal{E}}_{p}\right]=P\left[\operatorname{tr} \mathbf{Y}^{*} \overline{\mathbf{W}} \mathbf{Y}>0\right]
$$

where $\overline{\mathbf{W}}=(M-p)^{-1} \mathbf{P}_{\mathbf{U}_{0}}-p^{-1} \mathbf{P}_{\mathbf{U}_{p}}$. On the other hand, when the signal subspace eigenvalues are dispersed, it is reasonable to associate a subspace swap with the event $C$. Why? Because in this case it is more likely that the average energy in the orthogonal subspace will exceed $\mathcal{E}_{p p}$ than $\overline{\mathcal{E}}_{p}$. Therefore, we choose the event $C$ as the better bounding event. Then the probability of a subspace swap is bounded below by

$$
P[A] \geq P[C]=P\left[\overline{\mathcal{E}}_{0}>\overline{\mathcal{E}}_{p p}\right]=P\left[\operatorname{tr} \mathbf{Y}^{*} \mathbf{W} \mathbf{Y}>0\right]
$$

By using our representation for $\mathbf{Y}$, we may write tr $\mathbf{Y}^{*} \mathbf{W} \mathbf{Y}$ (or $\operatorname{tr} \mathbf{Y}^{*} \overline{\mathbf{W}} \mathbf{Y}$ ) as

$$
\operatorname{tr} \mathbf{Y}^{*} \mathbf{W Y}=\sum_{i=1}^{N} \sum_{j=1}^{N} y_{i}^{*} y_{j} \operatorname{tr} \mathbf{D}_{i}^{*} \mathbf{W} \mathbf{D}_{j}=\mathbf{y}^{*} \mathbf{S y}
$$

where $\mathbf{S}=\mathbf{S}^{*}$ is the Hermitian matrix

$$
\begin{aligned}
\mathbf{S} & =\left\{s_{i j}\right\}=\left\{\operatorname{tr} \mathbf{D}_{i}^{*} \mathbf{W} \mathbf{D}_{j}\right\}=\mathbf{A}+j \mathbf{B}=\mathbf{A}^{T}-j \mathbf{B}^{T}=\mathbf{S}^{*} \\
\mathbf{A} & =\mathbf{A}^{T} ; \quad \mathbf{B}=-\mathbf{B}^{T} .
\end{aligned}
$$

The quadratic form $\mathbf{y}^{*} \mathrm{Sy}$ is fundamental to our analysis of subspace swap. It can be further simplified by noting that the real, Hermitian symmetric, quadratic form $\mathbf{y}^{*} \mathbf{S y}$ may be written as the real symmetric quadratic form

$$
\begin{aligned}
c & =\mathbf{y}^{*} \mathbf{S y}=\left(\mathbf{y}_{r}-j \mathbf{y}_{i}\right)^{T}(\mathbf{A}+j \mathbf{B})\left(\mathbf{y}_{r}+j \mathbf{y}_{i}\right) \\
& =\left(\begin{array}{ll}
\mathbf{y}_{r}^{T} & \mathbf{y}_{i}^{T}
\end{array}\right)\left[\begin{array}{cc}
\mathbf{A} & -\mathbf{B} \\
\mathbf{B} & \mathbf{A}
\end{array}\right]\left(\begin{array}{l}
\mathbf{y}_{r} \\
\mathbf{y}_{i}
\end{array}\right)=\mathbf{z}^{T} \mathbf{T z}
\end{aligned}
$$

where

$$
\mathbf{z}=\left(\mathbf{y}_{r}^{T} \mathbf{y}_{i}^{T}\right) ; \quad \mathbf{T}=\left[\begin{array}{cc}
\mathbf{A} & \mathbf{B}^{T} \\
\mathbf{B} & \mathbf{A}
\end{array}\right]
$$

This simplification allows us to analyze the Hermitian quadratic form $\mathbf{y}^{*} \mathrm{Sy}$ as the real quadratic form $\mathbf{z}^{T} \mathbf{T z}$. The distribution of $\mathbf{z}=\left[\begin{array}{l}\mathbf{y}_{r} \\ \mathbf{y}_{i}\end{array}\right]$ is multivariate normal $N[\mathbf{m}, \mathbf{R}]$ when $\mathbf{y}$ is multivariate normal. Its two moments are

$$
\begin{aligned}
& \mathbf{m}=E \mathbf{z}=\left[\begin{array}{l}
\operatorname{Re}[\mathbf{x}] \\
\operatorname{Im}[\mathbf{x}]
\end{array}\right] \\
& \mathbf{R}=E\left[\begin{array}{l}
\operatorname{Re}[\mathbf{n}] \\
\operatorname{Im}[\mathbf{n}]
\end{array}\right]\left[\operatorname{Re}[\mathbf{n}]^{T} \operatorname{Im}[\mathbf{n}]^{T}\right]=\left[\begin{array}{ll}
\mathbf{R}_{11} & \mathbf{R}_{12} \\
\mathbf{R}_{21} & \mathbf{R}_{22}
\end{array}\right] .
\end{aligned}
$$

With this multivariate distribution, we may write the characteristic function of $c=\mathbf{y}^{*} \mathrm{Sy}=\mathbf{z}^{T} \mathbf{T z}$ as

$$
\begin{aligned}
\Phi_{c}(j \omega)= & E[\exp (-j \omega c)] \\
\Phi_{c}(j \omega)= & \int_{-\infty}^{\infty} \frac{1}{(2 \pi)^{\frac{2 N}{2}}}(\operatorname{det} \mathbf{R})^{-1 / 2} \exp \left\{-j \omega \mathbf{z}^{T} \mathbf{T} \mathbf{z}\right. \\
& \left.-\frac{1}{2}\left[(\mathbf{z}-\mathbf{m})^{T} \mathbf{R}^{-1}(\mathbf{z}-\mathbf{m})\right]\right\} d \mathbf{z} .
\end{aligned}
$$

Since $\mathbf{R}^{-1}$ is positive definite and $\mathbf{T}$ is a real symmetric matrix, we know from the simultaneous diagonalization theorem [8] that there exits a nonsingular matrix $\mathbf{P}$ such that

$$
\begin{aligned}
\mathbf{P}^{T} \mathbf{R}^{-1} \mathbf{P} & =\mathbf{I} \\
\mathbf{P}^{T} \mathbf{T P} & =\mathbf{D}
\end{aligned}
$$

where $\mathbf{D}$ is a diagonal matrix, not necessarily nonsingular, of real values.

The determinant of the matrix $\left(\mathbf{R}^{-1}+2 j \omega \mathbf{T}\right)$ can be written as

$$
\begin{aligned}
\operatorname{Det}\left[\mathbf{R}^{-1}+2 j \omega \mathbf{T}\right] & =\frac{\operatorname{Det}\left[\mathbf{P}^{T} \mathbf{R}^{-1} \mathbf{P}+2 j \omega \mathbf{P}^{T} \mathbf{T} \mathbf{P}\right]}{\operatorname{Det}\left[\mathbf{P}^{T} \mathbf{P}\right]} \\
& =\frac{\operatorname{Det}[\mathbf{I}+2 j \omega \mathbf{D}]}{\operatorname{Det}\left[\mathbf{P}^{T} \mathbf{P}\right]} \\
& =\frac{\prod_{i=1}^{\operatorname{rank}(\mathbf{D})}\left(1+2 j \omega d_{i i}\right)}{\operatorname{Det}\left[\mathbf{P}^{T} \mathbf{P}\right]}
\end{aligned}
$$


Since the $d_{i i}$ are real, this determinant can never equal zero, and therefore $\left(\mathbf{R}^{-1}+2 j \omega \mathbf{T}\right)$ has an inverse. By completing the square, we can write the exponent of $(22)$ as

$$
\begin{aligned}
& -\frac{1}{2}\left[\mathbf{z}-\left(\mathbf{R}^{-1}+2 j \omega \mathbf{T}\right)^{-1} \mathbf{R}^{-1} \mathbf{m}\right]^{T}\left(\mathbf{R}^{-1}+2 j \omega \mathbf{T}\right) \\
& \times\left[\mathbf{z}-\left(\mathbf{R}^{-1}+2 j \omega \mathbf{T}\right)^{-1} \mathbf{R}^{-1} \mathbf{m}\right]-\frac{1}{2} \mathbf{m}^{T} \mathbf{R}^{-1} \mathbf{m} \\
& +\frac{1}{2} \mathbf{m}^{T} \mathbf{R}^{-1}\left(\mathbf{R}^{-1}+2 j \omega \mathbf{T}\right)^{-1} \mathbf{R}^{-1} \mathbf{m} .
\end{aligned}
$$

This makes the integral of (22) a normal integral, meaning that the characteristic function is

$$
\begin{aligned}
\Phi_{c}(j \omega)= & {\left[\frac{\operatorname{det}\left[\mathbf{R}^{-1}+2 j \omega \mathbf{T}\right]^{-1}}{\operatorname{det}[\mathbf{R}]}\right]^{1 / 2} } \\
& \times \exp \left\{-\frac{1}{2} \mathbf{m}^{T} \mathbf{R}^{-1} \mathbf{m}+\frac{1}{2} \mathbf{m}^{T} \mathbf{R}^{-1}\right. \\
& \left.\times\left(\mathbf{R}^{-1}+2 j \omega \mathbf{T}\right)^{-1} \mathbf{R}^{-1} \mathbf{m}\right\}
\end{aligned}
$$

or upon simplification

$$
\begin{aligned}
\Phi_{c}(j \omega)= & {\left[\frac{1}{\operatorname{det}[\mathbf{I}+2 j \omega \mathbf{R T}]}\right]^{1 / 2} } \\
& \times \exp \left\{-\frac{1}{2} \mathbf{m}^{T} \mathbf{R}^{-1}\left[\mathbf{I}-(\mathbf{I}+2 j \omega \mathbf{R T})^{-1}\right] \mathbf{m}\right\}
\end{aligned}
$$

When $\mathbf{R}_{y y}=\sigma^{2} \mathbf{I}$ (see (3)), then $\mathbf{R}=\frac{\sigma^{2}}{2} \mathbf{I}$. The characteristic function is then

$$
\begin{aligned}
\Phi_{c}(j \omega)= & {\left[\frac{1}{\operatorname{det}\left[\mathbf{I}+j \omega \sigma^{2} \mathbf{T}\right]}\right]^{1 / 2} } \\
& \times \exp \left\{-\frac{1}{\sigma^{2}} \mathbf{m}^{T}\left[\mathbf{I}-\left(\mathbf{I}+j \omega \sigma^{2} \mathbf{T}\right)^{-1}\right] \mathbf{m}\right\}
\end{aligned}
$$

Let us suppose the symmetric matrix $\mathbf{T}$ has eigenvalue decomposition given by

$$
\mathbf{T}=\mathbf{Q} \Lambda \mathbf{Q}^{T}
$$

where

$$
\mathbf{Q}^{T} \mathbf{Q}=\mathbf{Q Q}^{T}=\mathbf{I} ; \quad \Lambda=\operatorname{diag}\left(\lambda_{1}, \lambda_{2}, \ldots, \lambda_{2 N}\right) .
$$

Then, in the new coordinates, the characteristic function is

$$
\begin{aligned}
\Phi_{c}(j \omega)= & {\left[\frac{1}{\prod_{i=1}^{2 N}\left(1+j \omega \sigma^{2} \lambda_{i}\right)}\right]^{1 / 2} } \\
& \times \exp \left\{-j \omega \sum_{i=1}^{2 N} \frac{\lambda_{i} \gamma_{i}^{2}}{\left(1+j \omega \sigma^{2} \lambda_{i}\right)}\right\} \\
= & \prod_{i=1}^{2 N} \Phi_{c_{i}}(j \omega)
\end{aligned}
$$

where

$$
\Phi_{c_{i}}(j \omega)=\left[\frac{1}{\left(1+j \omega \sigma^{2} \lambda_{i}\right)}\right]^{1 / 2} \exp \left\{-j \omega \frac{\lambda_{i} \gamma_{i}^{2}}{\left(1+j \omega \sigma^{2} \lambda_{i}\right)}\right\}
$$

and $\gamma_{i}=\left(\mathbf{Q}^{T} \mathbf{m}\right)_{i}$ is a coefficient of $\mathbf{m}$ in the orthogonal coordinates $\mathbf{Q}$.

The characteristic function for $c$ tells the whole story: The random variable $c$, whose threshold crossing of zero constitutes a subspace swap, is distributed as the sum of $2 N$ independent random variables $c_{i}$. Each of these random variables, $c_{i}$, is a chi-squared random variable with noncentrality parameter $2 \gamma_{i}^{2} / \sigma^{2}$, scaled by $\lambda_{i} \frac{\sigma^{2}}{2}$. That is

$$
\begin{aligned}
c=\sum_{i=1}^{2 N} c_{i} & =\sum_{i=1}^{2 N} \frac{\lambda_{i} \sigma^{2}}{2} c_{i}^{\prime} \\
c_{i}^{\prime}: & \chi_{1}^{2}\left(\frac{2 \gamma_{i}^{2}}{\sigma^{2}}\right) .
\end{aligned}
$$

This constitutes an exact statistical characterization of the subspace swap events $B$ and $C$.

For numerical computation of probabilities, we simply inverse Fourier-transform the characteristic function for $c$ :

$$
f(c)=F^{-1}\left[\Phi_{c}(j \omega)\right]
$$

Then, we numerically integrate this density for $c>0$ to obtain

$$
P\left[\overline{\mathcal{E}}_{0}>\mathcal{E}_{p p}\right] \text {. }
$$

In order to compute $P\left[\overline{\mathcal{E}}_{0}>\overline{\mathcal{E}}_{p}\right]$ we simply replace $\mathbf{W}$ by $\overline{\mathbf{W}}$ in (17).

\section{RESULTS}

In this section, we apply the theoretical results derived above to an example presented by TKV (Example 2, pg. 312 in [2]). In this example, the modal signal is the sum of two exponentials:

$$
s_{k}=e^{j \frac{\pi}{18} k}+e^{j \frac{41 \pi}{90} k}, \quad k=1,2, \ldots, 25 .
$$

The observation is a noisy version of $s_{k}$, namely $y_{k}=s_{k}+n_{k}$, where the noise is assumed to be the standard normal complex white noise with independent real and imaginary components of respective variances $\sigma^{2} / 2$. The signal-to-noise ratio is computed from the ratio of the total signal power to the total noise power:

$$
\mathrm{SNR}=10 \log _{10}\left(\frac{\text { Total Signal Power }}{N \sigma^{2}}\right)=10 \log _{10}\left(\frac{2}{\sigma^{2}}\right) .
$$

This is also the per sample SNR or the input SNR.

We use an overfitting parameter, $m=7$, to form the necessary Hankel matrices of size $19 \times 7$. Since the signal components are orthogonal with unit powers, the signal subspace eigenvalues are clustered. Therefore, we use the condition that $\overline{\mathcal{E}}_{0}$ be greater than $\overline{\mathcal{E}}_{p}$ to bound the probability of a subspace swap. Using (30), we generate samples of the characteristic function at a sufficiently high sampling rate. These samples are inverted using an FFT to get samples of the probability density function. We then integrate the positive tail of the probability density function to get the desired bounding probability in accordance with (33).

In Fig. 2, we present the lower bound of the probability of a subspace swap as a function of the SNR. For comparison, we 


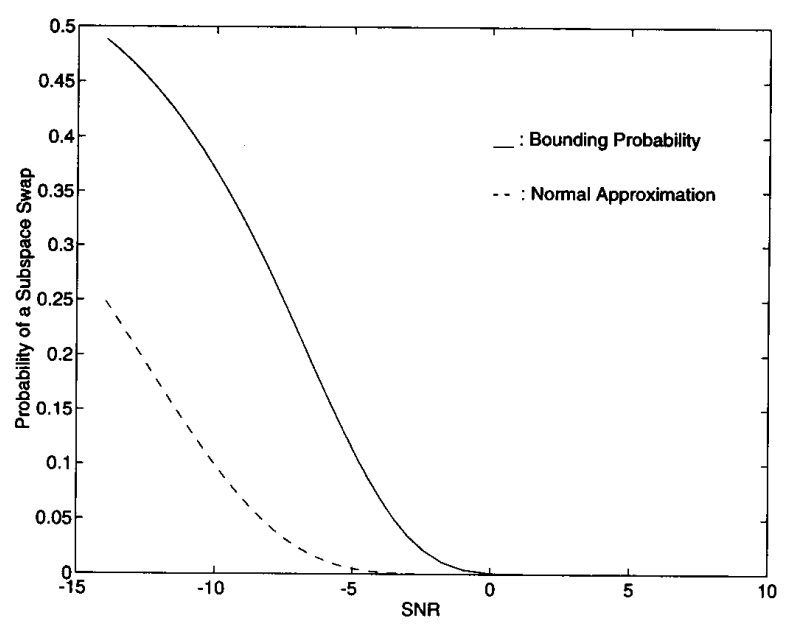

Fig. 2. Comparison of swap probabilities computed using the exact density and the normal approximation.

also present the approximate probability of a subspace swap, which is computed by assuming that the quadratic form in (20) is normally distributed. The mean and the variance of the approximating normal random variable are computed by noting that the random variable $c$ (see 32) is the sum of $2 N$ random variables $c_{i}$. The mean and variance of each of the $c_{i}$ 's is

$$
\begin{aligned}
E\left[c_{i}\right] & =\lambda_{i} \gamma_{i}^{2}+\frac{\sigma^{2}}{2} \lambda_{i} \\
\operatorname{Var}\left[c_{i}\right] & =\frac{1}{2}\left[\sigma^{2} \lambda_{i}^{2} \gamma_{i}^{2}+\sigma^{4} \lambda_{i}^{2}\right] .
\end{aligned}
$$

Therefore, the mean and variance of $c$ are

$$
\begin{aligned}
E[c] & =\sum_{i=1}^{2 N} \lambda_{i} \gamma_{i}^{2}+\frac{\sigma^{2}}{2} \lambda_{i} \\
\operatorname{Var}[c] & =\frac{1}{2} \sum_{i=1}^{2 N}\left[\sigma^{2} \lambda_{i}^{2} \gamma_{i}^{2}+\sigma^{4} \lambda_{i}^{2}\right] .
\end{aligned}
$$

The normal approximation presented in Fig. 2 will be different from the approximation obtained by TKV because they use an approximate condition for a subspace swap, while we use a sufficient condition. We note that the true density provides a tighter lower bound than the normal approximation.

In their analysis, TKV defined the total variance, $\sigma_{T}^{2}$, for estimating the modal parameters in terms of the Cramer-Rao bounds and swap probability as

$$
\sigma_{T}^{2}=\sigma_{0}^{2}\left(P_{s}\right)+\sigma_{\mathrm{CR}}^{2}\left(1-P_{s}\right)
$$

where

$$
\begin{aligned}
\sigma_{T}^{2} & \text { total variance } \\
\sigma_{0}^{2} & \text { variance when a swap has occurred }=\frac{180^{2}}{3} \\
P_{s} & \text { probability of a subspace swap } \\
\sigma_{\mathrm{CR}}^{2} & \text { variance given by the Cramer-Rao Bound. }
\end{aligned}
$$

Using numerical experiments, TKV were able to show that the relationship in (38) well models the threshold effect. That

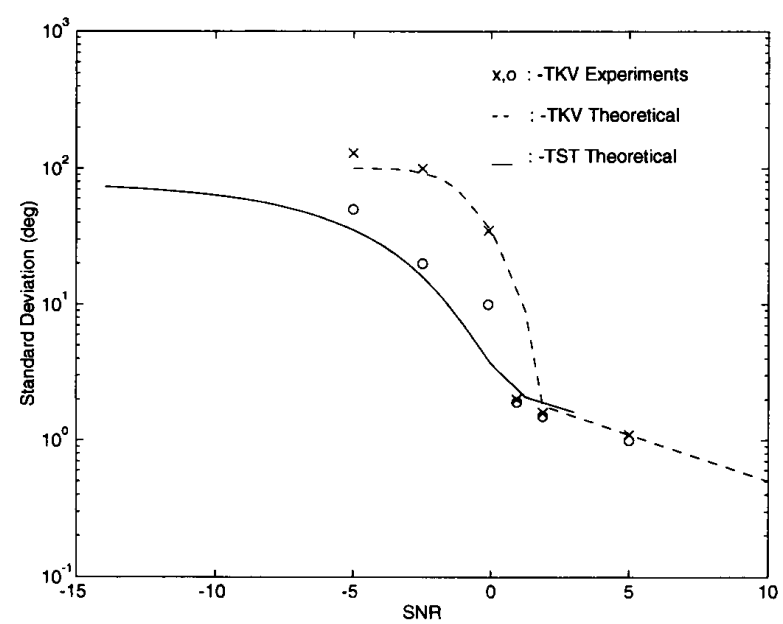

Fig. 3. Comparison of the square root of the total variance (38) computed using the approximate probability of a subspace swap (TKV) and the bounding probability $P\left[\overline{\mathcal{E}}_{0}>\overline{\mathcal{E}}_{p}\right]$. Experimental results of TKV are superimposed.

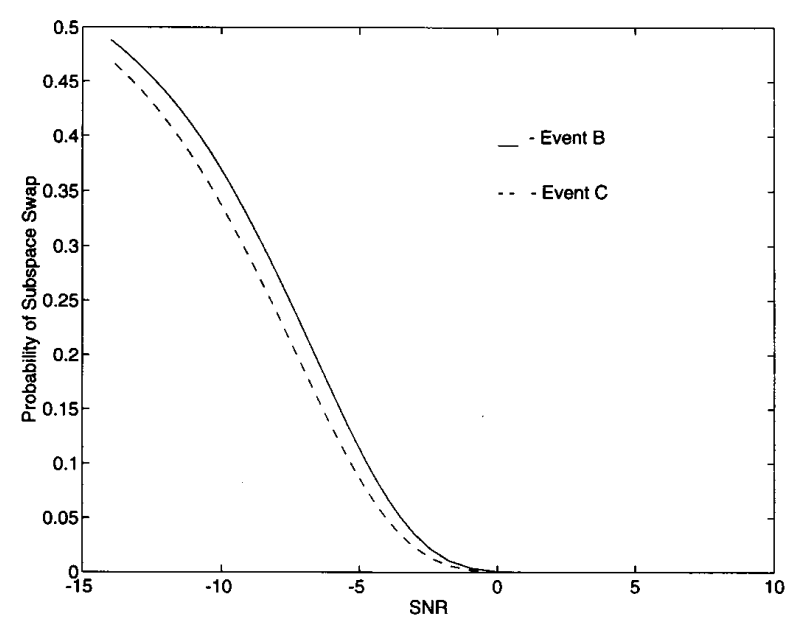

Fig. 4. Comparison of the sufficient conditions for a subspace swap: Event $B$-average energy in orthogonal subspace exceeds average energy in signal subspace; Event C-average energy in orthogonal subspace exceeds energy in a priori least dominant signal mode.

result is presented in Fig. 3. We use the bounding probability from our analysis in place of the approximate probability to compute the total variance in (38), and the resulting curve is presented in Fig. 3, superimposed on the results of TKV. We note that the total variance computed using the bounding probability can be used to predict the onset of threshold for the identification of both modes.

We argued in Section III that the appropriate bounding event for this problem is the one that compares the average energy in the orthogonal subspace to the average energy in the signal subspace (event $B$ ). In Fig. 4, we compare the probabilities computed using event $B$ and event $C$. We note that, as expected, the probability computed using event $B$ produces a tighter bound than the probability computed using event $C$.

Changing the overfit parameter $m$ has an impact on the probability of a subspace swap. For example, if $m$ is decreased, 


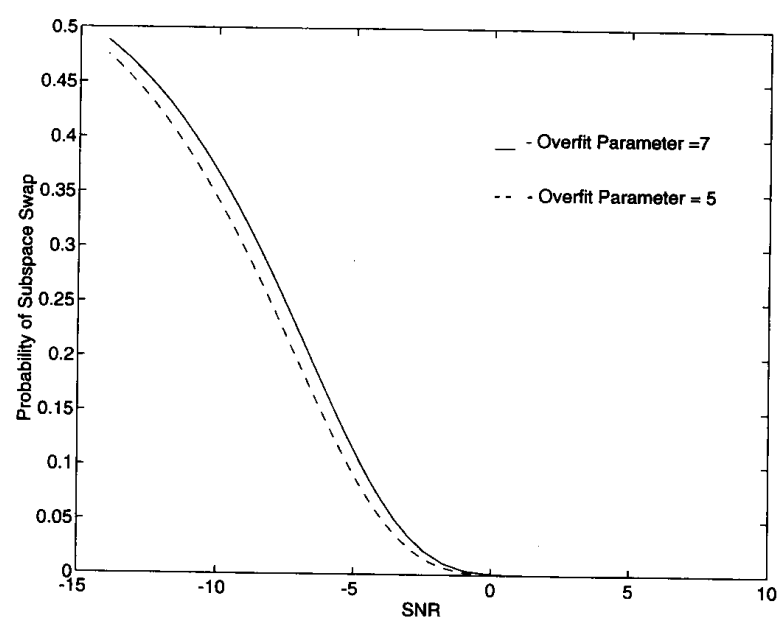

Fig. 5. Impact of changing the overfit parameter $m$.

then $M=N-m+1$ is increased, and the energy in the orthogonal subspace is distributed over a larger number of modes. This distribution of energy tends to reduce the chance of the energy in one of those modes exceeding the energy in the signal subspace. We conclude that decreasing $m$ tends to reduce the probability of a subspace swap and this is illustrated in Fig. 5.

Next, we analyze an example where the signal subspace singular values are dispersed. In this example, the modal signal is a sum of two exponentials and is given by

$$
s_{k}=e^{j \omega(0.5) k}+e^{j \omega(0.52) k}, \quad k=1,2 \ldots, 25 .
$$

In this case, the signal components are nearly colinear; therefore the signal subspace resolves signal energy into a dominant component along a principal eigenvector and a subdominant component along a perpendicular eigenvector. Therefore, we bound the probability of a subspace swap with the condition $\overline{\mathcal{E}}_{0}>\mathcal{E}_{p p}$ (event $C$ ). The observation is a noisy version of $s_{k}$, namely $y_{k}=s_{k}+n_{k}$ where the noise $n_{k}$ is assumed to be the standard normal complex white noise with independent real and imaginary components of respective variances $\sigma^{2} / 2$.

Once again, we use an overfitting parameter, $m=7$, to form the necessary Hankel matrices of size $19 \times 7$ and generate the bounding probabilities as a function of SNR. These bounding probabilities are plotted as a function of SNR in Fig. 6. We conclude that, as claimed, event $C$ produces a tighter-bounding probability than is obtained using event $B$. Finally, the total variance for estimating the angle of the mode was computed using (38), and the resulting curve is presented in Fig. 7. We also present, in Fig. 7, the standard deviation of the error in experimentally estimating the angle of the mode. Note the experimental results follow the theoretical curve, suggesting that the bounding probabilities can be used to predict the onset of threshold in performance.

\section{CONCLUSION}

We have extended the work of Tufts, Kot, and Vaccaro for the analysis of threshold effects in linear prediction methods

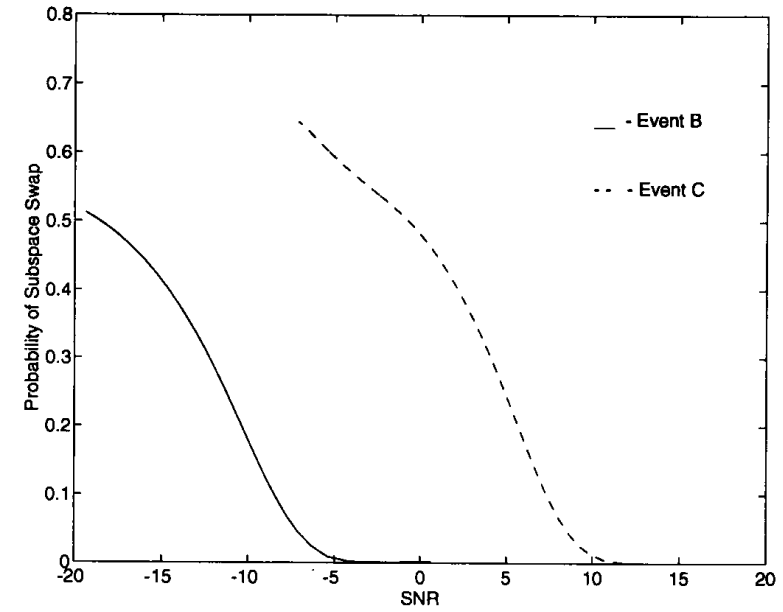

Fig. 6. Comparison of the sufficient conditions for a subspace swap: Event $B$-average energy in orthogonal subspace exceeds average energy in signal subspace; Event C-average energy in orthogonal subspace exceeds energy in a priori least dominant signal mode.

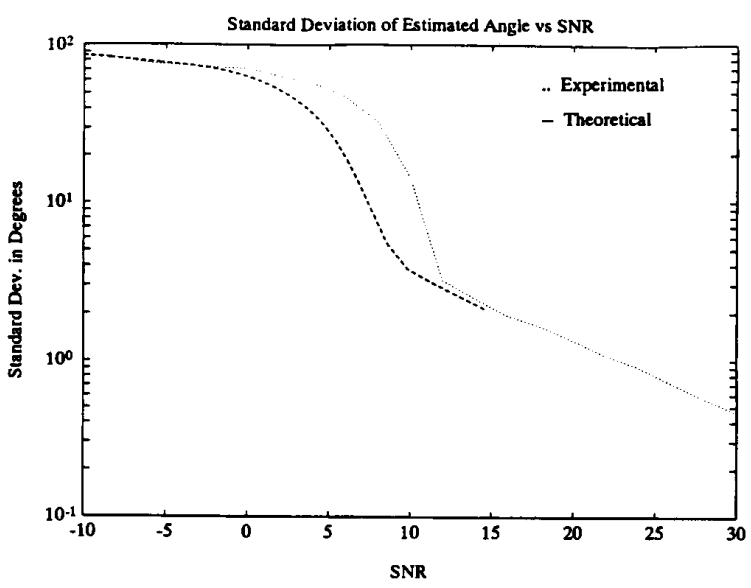

Fig. 7. Comparison of the square root of the total variance (38) with experimental results.

that use the SVD for rank reduction. We have defined two sufficient conditions for the onset of a subspace swap, as opposed to an approximate condition, and demonstrated the conditions under which each is applicable. We have computed the exact distributions, as opposed to normal approximations, for these sufficient conditions and as a result we have been able to lower bound the probability of a subspace swap. Finally, we have validated that the total variance, as defined in (38) - though not a lower bound theoretically - can be used to explain the onset of threshold in subspace methods. Future research is centered around using these results to study the order selection problem.

\section{REFERENCES}

[1] D. W. Tufts, A. C. Kot, and R. J. Vaccaro "The analysis of threshold behavior of SVD-based algorithms," in Proc. XXIst Annu. Asilomar Conf. Signals. Syst. Comput., Nov. 1987. 
[2] _ "The threshold effect in signal processing algorithms which use an estimated subspace," in SVD and Signal Processing, II: Algorithms, Analysis and Applications (R. Vaccaro, Ed.). New York: Elsevier, 1991.

[3] R. Kumaresan, "Estimating the parameters of exponentially damped sinusoidal signals in noise," Ph.D. dissertation, University of Rhode Island, Kingston, RI, Aug. 1982.

[4] R. Kumaresan and D. W. Tufts, "Estimating the parameters of exponentially damped sinusoids and pole-zero modeling in noise," IEEE Trans Acoust., Speech, Signal Processing, vol. 30, no. 6, pp. 833-840, Dec. 1982.

[5] D. W. Tufts and R. Kumaresan, "Frequency estimation of multiple sinusoids: Making linear prediction work like maximum likelihood," Proc. IEEE, vol. 70, pp. 975-990, Mar. 1983.

[6] R. Kumaresan, D. Tufts, and L. L. Scharf, "A prony method for noisy data: Choosing the signal components and selecting the order in exponential signal models," Proc. IEEE, vol. 72, no. 2, pp. 230-233, Feb. 1984.

[7] A. Paulraj, R. Roy, and T. Kailath, "Estimation of signal parameters via rotational invariance techniques-ESPRIT," in Proc. XIXth Asilomar Conf. Circuits, Syst. Comput., Pacific Grove, CA, Nov. 1985, pp. 83-89.

[8] B. Noble, Applied Linear Algebra. Englewood Cliffs, NJ: PrenticeHall, 1977.

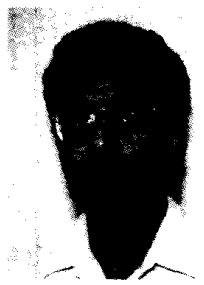

John K. Thomas received the B.S.E.E. degre (Hons.) in 1985 and the M.S.E.E. degree in 1987, both from the University of Arkansas, Fayetteville. Since 1992, he has been a Ph.D. student in electrical engineering at the University of Colorado, Boulder, working in the area of statistical signal processing.

In 1986 , he was employed as a consultant at the Jet Propulsion Laboratory to work on the Shuttle Imaging Radar (SIR-B) radargrammetry experiment. From 1987 to 1992, he worked at Vexcel Corporation in Boulder, where he was involved in

developing image/signal processing algorithms for radar signal analysis and reconstruction.

Mr. Thomas is a cofounder of Data Fusion Corporation and is a member of Eta Kappa $\mathrm{Nu}$ and Tau Beta Pi.

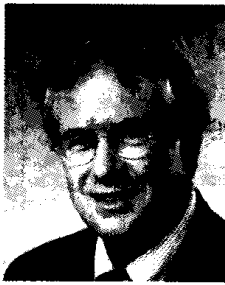

Louis L. Scharf (S'67-M'69-SM'77-F'86) received the Ph.D. degree in electrical engineering from the University of Washington, Seattle, in 1969.

From 1969 to 1971 , he was a member of the Technical Staff at Honeywell's Marine Systems Center in Seattle. He was a faculty member at Colorado State University, Fort Collins, from 1971 to 1981 , where he last served as a Professor of Electrical Engineering and Statistics. From 1982 to 1985, he was a Professor and the Chairman of Electrical Engineering at the University of Rhode Island, Kingston. He is currently a Professor of Electrical and Computer Engineering at the University of Colorado, Boulder, where he teaches and conducts research in signal processing. In 1974, he was a Visiting Associate Professor at Duke Univeristy, Durham, NC. In 1977, he was at the University of South Paris, Orsay, where he was a member of the Technical Staff in the SNRS Laboratorie des Signaux et Systemes, Gif-sur-Yvette. In 1981, he was a Visiting Professor at the Ecole Nationale Superieure des Telecommunications, Paris. In 1992 , he was a Visiting Professor at Institut Eurecom, Sophia-Antipolis, France. He has served as a consultant to Hewlett-Packard, Honeywell, Inc., the Applied Physics Labs-Seattle, the Research Triangle Institute, Green Mountain Geophysics, and Ball Aerospace Corporation.

Dr. Scharf is a past member of the ASSP ADCOM and the Editorial Board of Signal Processing. He is a past Assocate Editor for the IEEE Transactions on Acoustics, Speech, and Signal Processing. He was the Technical Program Chairman for the 1980 International Conference on ASSP and is a member of Eta Kappa $\mathrm{Nu}$.

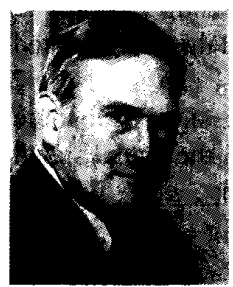

Donald W. Tufts (S'58-M'61-SM'78-F'82) was born March 5, 1933 in Yonkers, NY. He received the B.A. degree in mathematics from Williams College, Williamstown, MA, in 1955. In 1957, 1958, and 1960, he received the S.B., S.M., and Sc.D. degrees from the Massachussetts Institute of Technology, Cambridge, where he was a Bell Telephone Laboratories Fellow.

From 1960 to 1967 , he was at Harvard University, first as a Research Fellow and Lecturer and later as Assistant Professor of Applied Mathematics. From 1967 to the present, he has been a Professor of Electrical Engineering at the University of Rhode Island, Kingston. He has been a consultant to Bell Telephone Laboratories, Sanders Associates, Inc., and to other companies. 